\title{
Suppression of Specular Reflections by Metasurface with Engineered Nonuniform Distribution of Reflection Phase
}

\author{
Xin Mi Yang, ${ }^{1,2}$ Ge Lan Jiang, ${ }^{1}$ Xue Guan Liu, ${ }^{1}$ and Cheng Xiang Weng ${ }^{3}$ \\ ${ }^{1}$ School of Electronics and Information Engineering, Soochow University, Suzhou 215006, China \\ ${ }^{2}$ State Key Laboratory of Millimeter Waves, Southeast University, Nanjing 210096, China \\ ${ }^{3}$ Electronic Countermeasure Laboratory, Air Force Early Warning Academy, Wuhan 430019, China
}

Correspondence should be addressed to Xin Mi Yang; yangxinmi@suda.edu.cn

Received 9 November 2014; Accepted 8 January 2015

Academic Editor: Sanming Hu

Copyright (C) 2015 Xin Mi Yang et al. This is an open access article distributed under the Creative Commons Attribution License, which permits unrestricted use, distribution, and reproduction in any medium, provided the original work is properly cited.

\begin{abstract}
We make preliminary investigations on a new approach to reducing radar cross section (RCS) of conducting objects. This approach employs novel planar metasurfaces characterizing nonuniform distribution of reflection phase. The operation principle of this approach and the design rule of the associated metasurfaces are explained using a simplified theoretical model. We then present a design example of such metasurfaces, in which three-layer stacked square patches with variable sizes are utilized as the reflecting elements. The proposed RCS-reduction approach is verified by both numerical simulations and measurements on the example, under the assumption of normal plane wave incidence. It is observed that, in a fairly wide frequency band (from 3.6 to $5.5 \mathrm{GHz}$ ), the presented example is capable of suppressing the specular reflections of conducting plates significantly (by more than $7 \mathrm{~dB}$ ) for two orthogonal incident polarizations.
\end{abstract}

\section{Introduction}

Reduction of target radar cross section (RCS) for military or civilian applications has been a subject of extensive studies in both scientific and engineering communities for decades. The existing RCS-reduction strategies can be classified into two categories. One is decreasing or even canceling the scattered energy and the other is reshaping the scattering pattern. Traditionally, the radar absorbing material (RAM) is adopted for the first category while the second category is usually achieved through shaping of target [1-4]. Recently, great efforts have been made for the sake of improving the traditional RAM technology. For instance, reduced RAM thickness has been achieved by employing artificial magnetic conductor (AMC) or reactive impedance ground as the backing panel of RAM [5-7]. It is worth mentioning that the rapidly growing research field of metamaterials has contributed a completely new strategy called invisibility cloak $[8,9]$, which can be grouped into the first category. The invisibility cloak is a kind of inhomogeneous wrappage that could steer the incoming electromagnetic waves smoothly around the hidden object and return them to their original trajectory, making the object almost invisible (i.e., have no scattered field). New ideas have emerged regarding the second category of RCS-reduction techniques these years as well [10-14]. For example, it was proposed by Paquay et al. that, by combining AMC and perfect electrical conductor (PEC) cells in a chessboard-like configuration, the cancelation of reflections from these two kinds of cells would effectively reduce the specular reflections and hence the RCS of planar conducting plate [10]. In addition, some researchers have paid attention to metamaterial coatings with randomly distributed refractive indices or gradients of refractive index [11-13]. Such coatings are capable of suppressing remarkable lobes of conducting plates by creating diffuse reflections in front of these plates. In 2014, Wang et al. proposed a design of broadband and broad-angle low-scattering metasurface based on a hybrid optimization algorithm [14].

In this paper, we make further investigations on RCSreduction schemes characterizing redistribution of scattered energy, by making use of novel metasurfaces with nonuniformly distributed reflection phase. The rest of 


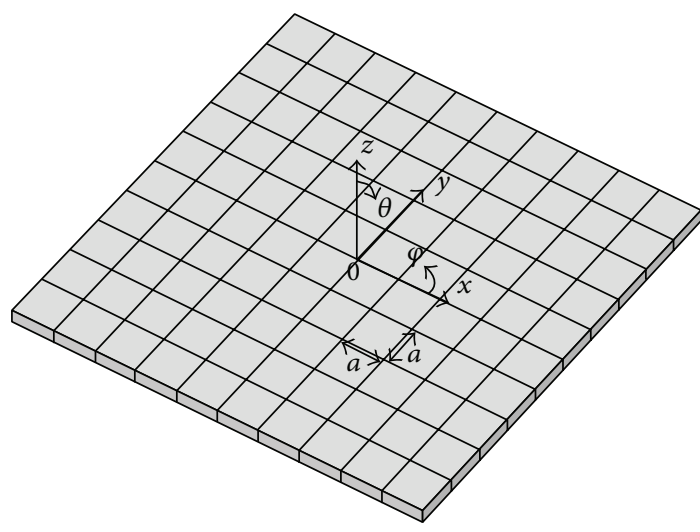

FIGURE 1: Sketch plot of a planar metasurface consists of $N_{x} \times N_{y}$ reflecting elements in square lattice.

the paper is organized as follows. In Section 2, a theoretical model for analyzing the backscattering performance of such metasurfaces is given and a fundamental design rule of the relevant nonuniform distribution of reflection phase is promoted for RCS-reduction in the specular direction. Section 3 introduces a practical implementation scheme of such metasurfaces through an example. Finally, Section 4 presents both the simulation and measurement results of the example, which confirms that metasurfaces with properly designed nonuniform phase distribution have the potential of suppressing specular reflections of planar conducting plates under normal incidence.

\section{Theory}

Metasurface with nonuniformly distributed reflection phase, which is referred to as nonuniform surface below for brevity, is artificial composite constituted by reflecting elements each of which reflects local incident ray back into space with certain phase shift. One or several geometrical parameters of these elements vary along the surface such that the reflection phase is nonuniformly distributed with respect to incident plane waves.

It is well known that directive radiation is closely related to equiphase surface perpendicular to the radiation direction. For planar conducting plate which has uniform reflection phase, the reflected field possesses a planar equiphase surface with respect to incident plane wave and hence the reflected energy is focused in specific direction (i.e., the specular direction). If the conducting plate is covered with certain composite structure to form a nonuniform surface, the equiphase surface will be no doubt disturbed and the incident plane wave will be reflected or scattered irregularly. Moreover, it is possible that the directional reradiation in the specular direction is remarkably suppressed or even eliminated, provided that the distribution of reflection phase is appropriately chosen.

For simplicity, consider the problem geometry shown in Figure 1, where a planar nonuniform surface is divided into $N_{x} \times N_{y}$ squares with lattice constant denoted by $a$. Each square represents a reflecting element which is assumed to totally reflect its local incident beam. The origin of coordinate is situated at the center of the plane defined by the surface. An infinite $y$-polarized plane wave is normally incident on the nonuniform surface and is given by

$$
\vec{E}_{I}=\dot{E}_{I} e^{j k_{0} z} \widehat{y}
$$

where $k_{0}$ is the wave number in free space. The local reflected field at each element can be estimated using the infinitely periodic array model and is supposed to be dominated by the fundamental copolarized Floquet mode (in this model, each element is analyzed by assuming local periodicity; i.e., each element is considered in an array environment with all the elements identical). Hence, the reflected electric field at the element numbered as $(l, s)\left(l=0,1,2, \ldots, N_{x}-1, s=\right.$ $\left.0,1,2, \ldots, N_{y}-1\right)$ can be written as

$$
\vec{E}_{R}(l, s)=\dot{E}_{I} e^{j \phi(l, s)} e^{-j k_{0} z} \widehat{y}
$$

where $\phi(l, s)$ is the reflection phase of the corresponding element. By applying the second principle of equivalence, the angular spectrum of plane waves for scattered field in the half-space $z>0$ can be expressed by the following Fourier transform [15]:

$$
\widetilde{E}_{R y}(u, v)=\int_{-\infty}^{\infty} \int_{-\infty}^{\infty} \dot{E}_{R y}(x, y) e^{j k_{0}(u x+v y)} d x d y,
$$

where $\dot{E}_{R y}(x, y)$ is the tangential component of the scattered electric field along the surface (i.e., the plane of $z=0$ ) and $u, v$ are variables related to the spherical coordinates $(\theta, \varphi)$ by $u=\sin \theta \cos \varphi$ and $v=\sin \theta \sin \varphi$. Note that the double integral in (3) is limited in the region of metasurface, because $\dot{E}_{R y}(x, y)$ is assumed to be zero outside of the surface. This assumption implies that the edge diffraction is ignored. Associating $\dot{E}_{R y}(x, y)$ with (2), the spectral function $\widetilde{E}_{R y}(u, v)$ is further derived as

$$
\widetilde{E}_{R y}(u, v)=a^{2} K S \cdot \sum_{s=0}^{N_{y}-1} \sum_{l=0}^{N_{x}-1} \dot{E}_{I} e^{j \phi(l, s)} e^{j k_{0} a(u l+v s)} .
$$

In the above formula,

$$
\begin{aligned}
& K=e^{-j\left(k_{0} a / 2\right)\left[u\left(N_{x}-1\right)+v\left(N_{y}-1\right)\right],} \\
& S=\mathrm{Sa}\left(\frac{k_{0} u a}{2}\right) \cdot \mathrm{Sa}\left(\frac{k_{0} v a}{2}\right),
\end{aligned}
$$

where $\mathrm{Sa}(x)=\sin (x) / x$, representing sample function.

For planar PEC plate with the same overall size, $\phi(l, s)$ should be replaced by $\pi$ and (5) is reduced to

$$
\widetilde{E}_{R y}(u, v)=-a^{2} K S \cdot \sum_{s=0}^{N_{y}-1} \sum_{l=0}^{N_{x}-1} \dot{E}_{I} e^{j k_{0} a(u l+v s)} .
$$

Obviously, the backscattering of PEC plate in the specular direction (i.e., $\theta=0$ ) would be effectively suppressed (by no less than $10 \mathrm{~dB}$ ) as long as the nonuniform distribution of 


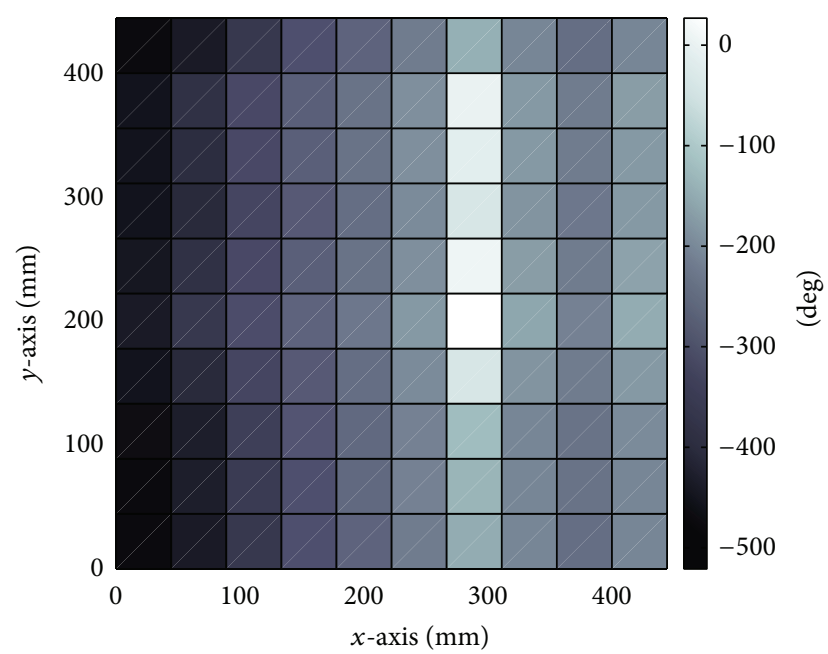

FIGURE 2: An instance of two-dimensional nonuniform distribution of reflection phase in degree, which is expected to significantly reduce the specular reflection of planar conducting plates.

reflection phase $\phi(l, s)$ satisfies the relation expressed by the following inequation:

$$
\left|\sum_{s=0}^{N_{y}-1} \sum_{l=0}^{N_{x}-1} e^{j \phi(l, s)}\right| \leq \sqrt{0.1} \cdot N_{x} N_{y} .
$$

Such a conclusion is in accordance with the antenna array theory and similar conclusions could be drawn for the cases of off-normal incidence.

As an example, consider a planar nonuniform surface containing $10 \times 10$ reflecting elements. It has the twodimensional phase distribution as shown in Figure 2. This distribution is generated by randomly selecting phases in the range [-504 deg, $40 \mathrm{deg}]$. With such a distribution, the amplitude of the double summation in (8) is calculated to be 23.15, which is much smaller than $N_{x} N_{y}=100$. According to the theoretical model presented above, the intensity of specular far-field scattering of the designed metasurface is $12.77 \mathrm{~dB}$ lower than that of naked conducting surface with the same overall size, when both surfaces are illuminated by $x$ - or $y$-polarized normally incident plane waves. We remark that the choice of the distribution in Figure 2 is rather arbitrary. In fact, the condition indicated by ( 8 ) is very weak and there are numerous possibilities for reflection-phase distributions which meet this condition. Therefore, wideband performance is in prospect even though the phase distribution varies violently in band. This is beneficial when broadband RCSreduction behavior is required, since the phase distribution of realistic nonuniform surface is usually frequency-dependent, as will be shown in the next section.

\section{Implementation of Nonuniform Surface}

Various radiators can be used as the reflecting elements of nonuniform surfaces. In particular, microstrip radiators such as metallic patches, dipoles, and loops are suitable for circumstances where conformality and compactness are required [16-19]. Figure 3 demonstrates a typical reflecting element in microstrip form, which characterizes three-layer stacked square patches of variable size. The stacked-patch element occupies an $a \times a$ square area in the $x-y$ plane and is backed with a conducting ground. The side lengths of patches on the bottom, middle, and top layers are denoted by $d, r_{1} d$, and $r_{2} d$, respectively, where $r_{1}$ and $r_{2}$ are proportional factors with $r_{2}<r_{1}<1$. Each of the three patches is supported by a piece of thin dielectric substrate which is also $a \times a$ in size. The patch thickness and substrate thickness for each layer are represented by $t_{m}$ and $t_{s}$, respectively. Moreover, Figure 3(b) indicates that every two neighboring layers (including the ground layer and the three patch layers) are separated by air, with the separation distances denoted by $h_{i}(i=1,2,3)$.

As is well known, the microstrip patch over a ground plane response resonantly to incident plane waves, leading to sensitive reflection phase with respect to frequency variations near resonance. The resonant frequency mainly depends on the side length of patch and the reflection phase within certain frequency band can be effectively tuned by varying the side length. By stacking multiple patches, a multiresonant behavior is obtained and the tuning range of reflection phase can be expanded greatly [20,21]. Moreover, stacking multiple patches is also helpful in enlarging the frequency band with linear response of reflection phase, which is critical in increasing the operational bandwidth of the nonuniform surface. The air separation employed under each patch layer further broadens the frequency band with sufficiently large tuning range of phase and results in a smoother and more linear phase variation versus side length $d$.

As an example, a parametric study has been made on the three-layer stacked-patch element with certain specifications. Each patch layer of the element under study is fabricated from F4B copper-clad laminate with copper thickness of $t_{m}=$ $0.035 \mathrm{~mm}$, substrate thickness of $t_{s}=0.43 \mathrm{~mm}$, and dielectric constant of $2.65-j 0.0053$. The element size, the relative sizes of the stacked patches, and the air separations are considered fixed ( $a=45 \mathrm{~mm}, r_{1}=0.68, r_{2}=0.5$, and $h_{1}=h_{2}=$ $h_{3}=4 \mathrm{~mm}$ ) and only the side length of bottom patch $d$ is the independently tunable variable. The parametric study is performed via numerical simulations using the commercial electromagnetic solver, CST Microwave Studio (MWS). Figure 3(c) shows the relation curve of simulated reflection phase at $4 \mathrm{GHz}$ against the dimension $d$ for the relevant stacked-patch elements. The reflection phase is associated with the fundamental copolarized Floquet mode with respect to normally incident plane waves polarized along the $x$ (or $y$-) axis, when periodic boundary conditions (PBCs) are assumed around the element in both the $x$ and $y$ directions. The reference plane just overlaps with the plane of top patch. It is seen from Figure 3(c) that the tuning range of reflection phase at $4 \mathrm{GHz}$ is greater than $500^{\circ}$.

Figure 4 illustrates a realistic nonuniform surface formed by arranging the three-layer stacked-patch elements in $a \times$ a square lattice. It is consistent with the model shown in Figure 1. The nonuniform distribution of reflection phase is achieved by varying the dimension $d$ and keeping all the other parameters (i.e., $t_{s}, t_{m}, r_{1}, r_{2}$, and $h_{i}$ ) constant in the surface. We remark that the structure in Figure 4 is quite similar to 


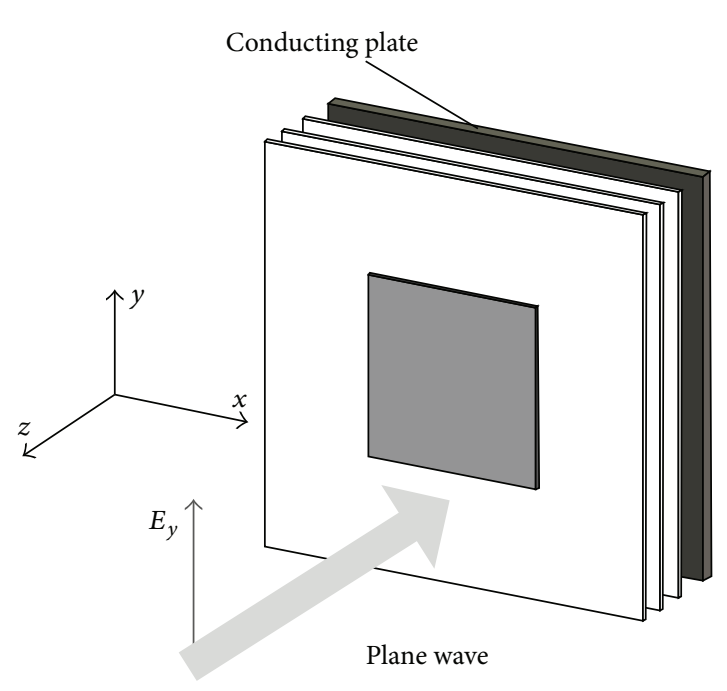

(a)

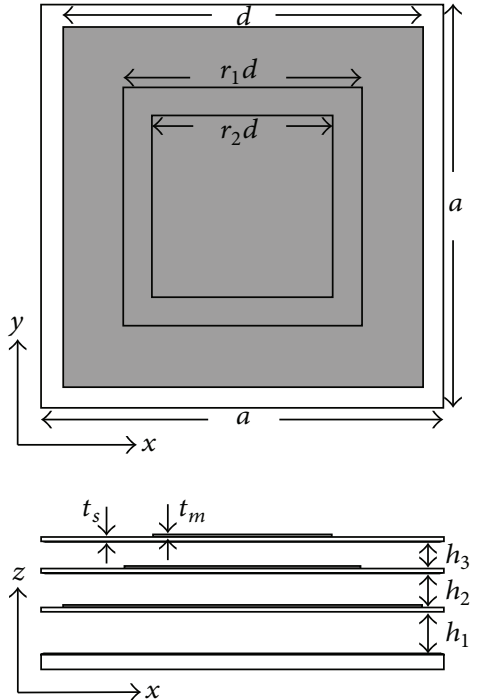

(b)

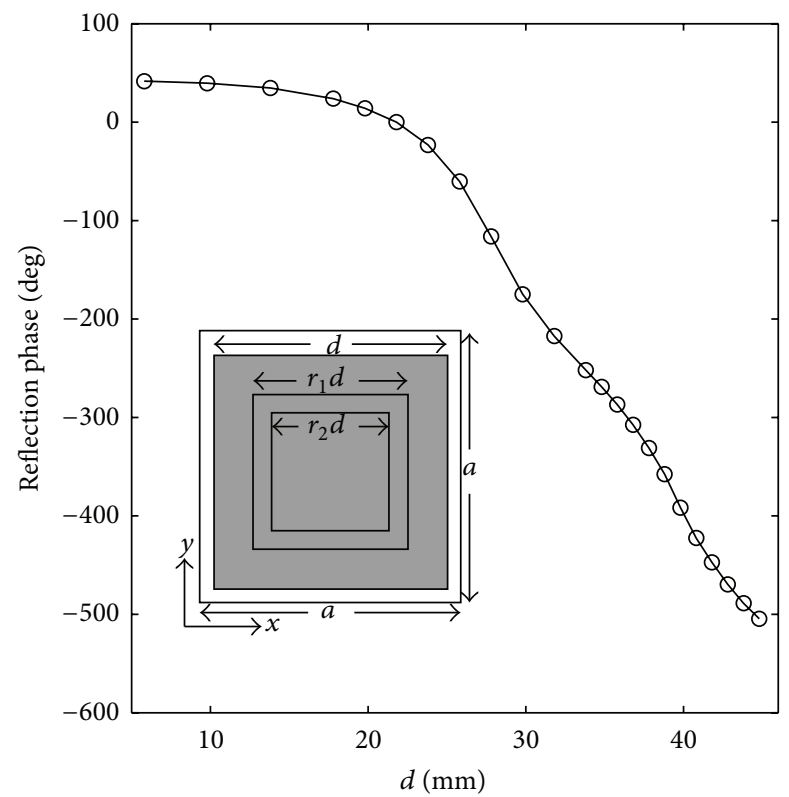

(c)

FIGURE 3: Reflecting element characterizing three-layer stacked square patches of variable size. (a) Oblique view. (b) Elevation view and side view. In the elevation view, the second and third substrate layers are omitted to show all the three patch layers clearly. (c) Typical design curve relating reflection phase at $4 \mathrm{GHz}$ with the dimension $d$ for the stacked-patch elements with certain specifications.

that of microstrip reflectarray [19]. However, the traditional reflectarray should be excited by certain feed antenna and is designed to produce directional reradiation, while the nonuniform surface proposed here has no feed antenna and is designed to destroy the directional reflection with respect to incoming plane waves.

An instance of nonuniform surface based on the stackedpatch elements has been designed and the corresponding fabricated sample is shown in Figure 5(a). This instance comprises $10 \times 10$ elements, which are characterized by the specifications mentioned above. Hence, the total thickness (from the top patch to the ground plane) of the instance is about $13.3 \mathrm{~mm}$. The instance was designed to possess the two-dimensional phase distribution shown in Figure 2 at $4 \mathrm{GHz}$. Given this phase distribution, the dimension $d$ of all the constituent elements for the nonuniform surface was determined using the design curve shown in Figure 3(c). The final distribution of dimension $d$ is shown in Figure 5(b). It should be remarked that the phase distribution here avoids drastic change of patch size between neighboring elements so that the periodic array model for element evaluation mentioned above maintains enough accuracy. 


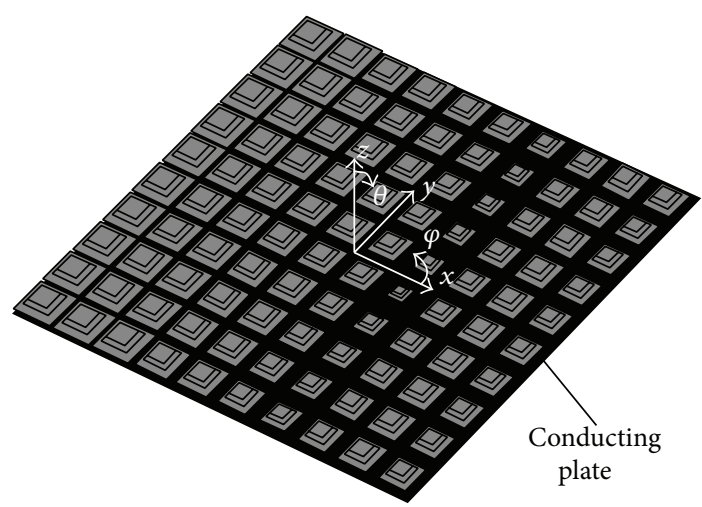

FIGURE 4: Nonuniform surface consisting of three layers of square patches with variable size above a conducting ground. All the substrate layers are set transparent so that the ground layer and the bottom and middle patch layers could be shown.

Due to the frequency-dependent phase response of the stacked-patch element, the phase distribution of the nonuniform surface changes with frequency. However, for our presented instance, the nonuniform feature of the phase distribution within a sufficiently large range remains over a fairly wide frequency band, that is, from about $3 \mathrm{GHz}$ to $6 \mathrm{GHz}$. Figure 6 demonstrates the specular far-field reflectance for the instance in this band (circle). This reflectance curve, which assumes normal incidence and $x$ - or $y$-polarization, is normalized to that of naked conducting surface and is calculated from the frequency-dependent phase distribution using the aforementioned theoretical model. Remarkable suppression of specular reflection throughout the band is observed from Figure 6.

\section{Simulation and Measurement Results}

In order to verify the nondirectional reflection property of the nonuniform surface, full-wave simulations are conducted for the instance presented above, with the aid of CST MWS. Both the $x$ - and $y$-polarized plane waves with normal incidence are considered in the simulations. For both polarizations, the simulated specular far-field reflectance curves, normalized to that of naked conducting surface, are also shown in Figure 6 (solid and dashed lines). As is expected, these two curves are in good consistency with the theoretical curve (circle). Figure 6 also indicates that the two simulated curves are slightly different from each other. This is because the nonuniform surface as instance has no rotational symmetry with respect to its central normal. Our theoretical model ignores this asymmetry and offers the same calculated reflectance results for both polarizations. However, such an ignorance or approximation does not prevent the model from providing us with acceptable predictions of backscattering performance of nonuniform surfaces. A further inspection on the simulated backscattering patterns of both the nonuniform surface and the naked conducting surface reveals that the suppression of specular reflections is achieved through unequally reallocating most of the scattered energy to various off-specular

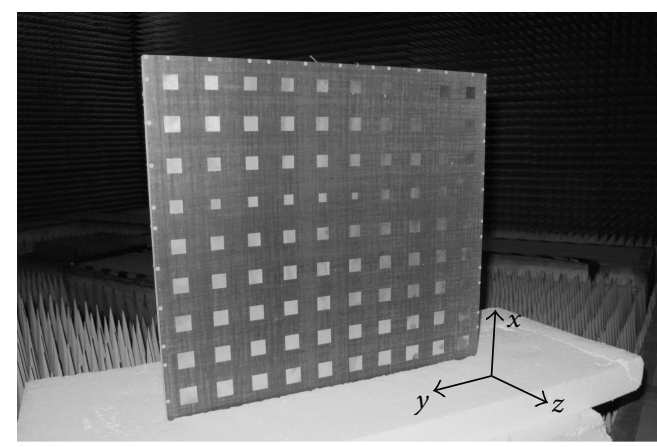

(a)

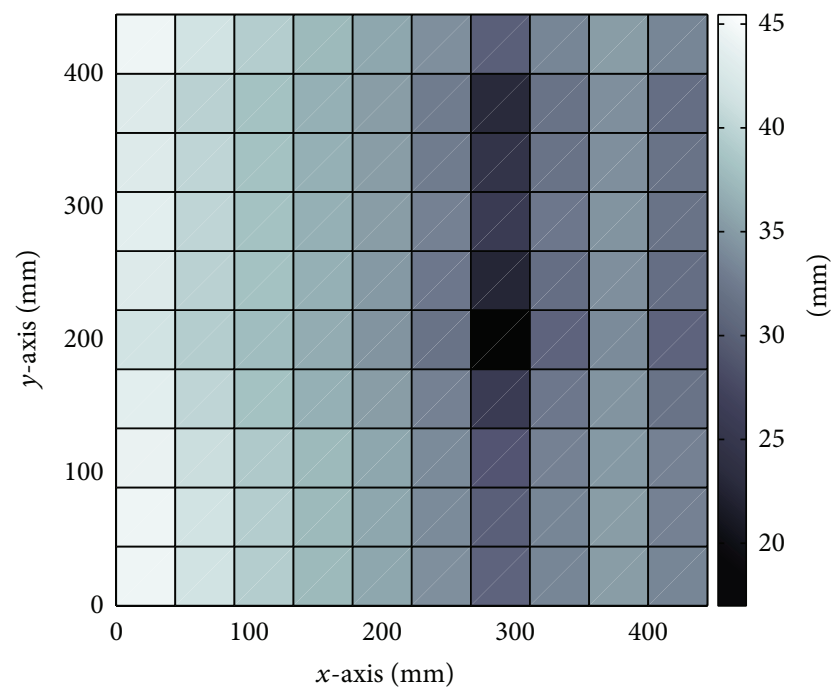

(b)

FIGURE 5: (a) Photograph of a nonuniform surface sample composed of three-layer stacked-patch elements. Note that the bottom row of elements is buried in foams and can not be seen. (b) Twodimensional distribution of dimension $d$ for patches on the bottom layer (unit: millimeters).

directions in the $z>0$ half-space (i.e., $0 \leq \theta<90$ ). As an example, Figure 7 compares the simulated backscattering patterns between the nonuniform surface and the naked conducting surface at $4 \mathrm{GHz}$ for the $y$-polarized normal incidence.

Backscattering measurements have also been conducted concerning the nonuniform surface sample shown in Figure 5. The measurements consist of two stages which employ almost the same experimental setup. Figure 8 shows the experimental setup for the first stage, where a linearly polarized high-gain horn antenna is placed right in front of the nonuniform surface sample with a distance of 3 meters and the central normal of the sample surface is aligned with the symmetry axis of the horn aperture. The horn antenna is also connected to a single port of an Agilent N5230C vector network analyzer (VNA) through a flexible coaxial cable. The whole setup is situated in an anechoic chamber to minimize environmental interferences. During measurement, the VNA provides source microwave signals for the antenna and the main beam emitted from the horn antenna impinges on 


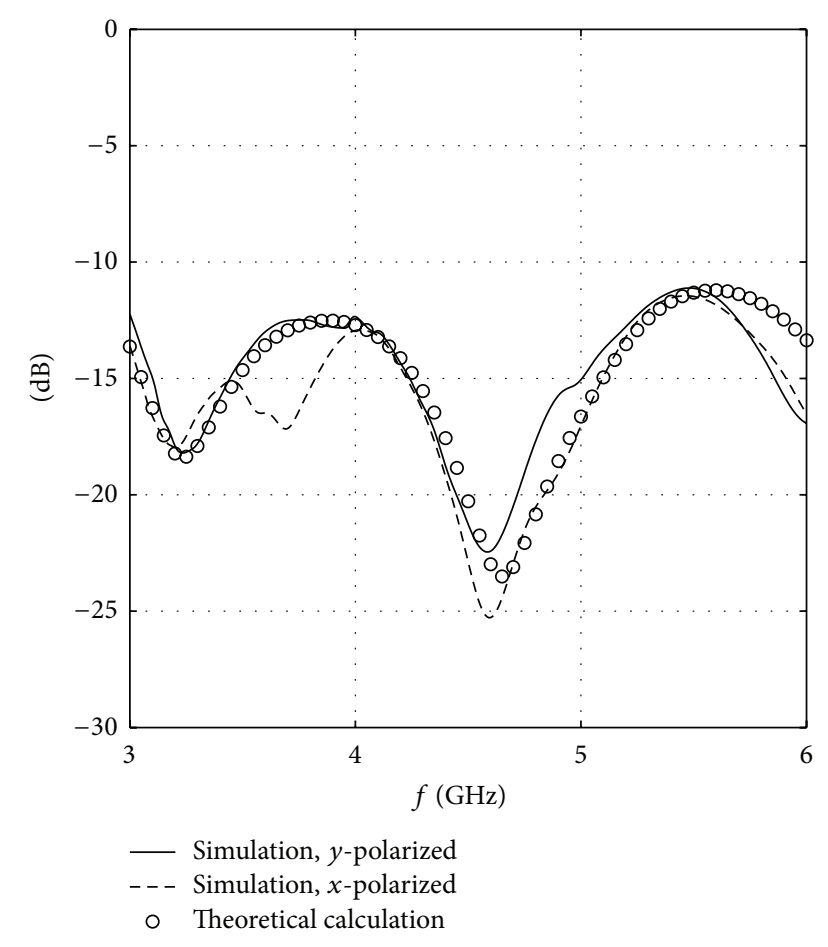

FIGURE 6: Theoretical and simulated results of the normalized specular far-field reflectance of the nonuniform surface as instance under normal incidence.

the sample surface. The back-scattered or reflected power from the sample is received by the same horn and then detected by the VNA. Note that the power reflected by the horn itself also enters the VNA in the meantime. By applying the gated-reflect-line (GRL) calibration technique provided by the time-domain analysis kit installed in the VNA, the influence of the power reflected by the horn is removed and the resultant reflection coefficient data read from the VNA is only related to the reflection from the sample. Two cases of incident beams are considered in the first stage. For the first case, the horn is arranged to allow a $y$-polarized incident beam with respect to the sample shown in Figure 5. By simply rotating the sample $90^{\circ}$ around its central normal, the other case of incident beam with $x$-polarization can be achieved afterwards. The reflection coefficient data in decibels read from the VNA are recorded for both cases. As to the second stage of measurement, the nonuniform surface sample is replaced by an ordinary square metallic plate which possesses the same size as the sample. The reflection coefficient data in decibels given by the VNA at this stage is also recorded. The specular reflectance of the nonuniform surface sample normalized to that of the square metallic surface for both incident polarizations can then be obtained by subtracting the newly recorded data from those two sets of data measured at the first stage, respectively.

Figure 9 shows the experimental normalized specular reflectance of the nonuniform surface sample for both incident polarizations. It is observed that, in the presence of the sample, the directional specular reflection of the metallic

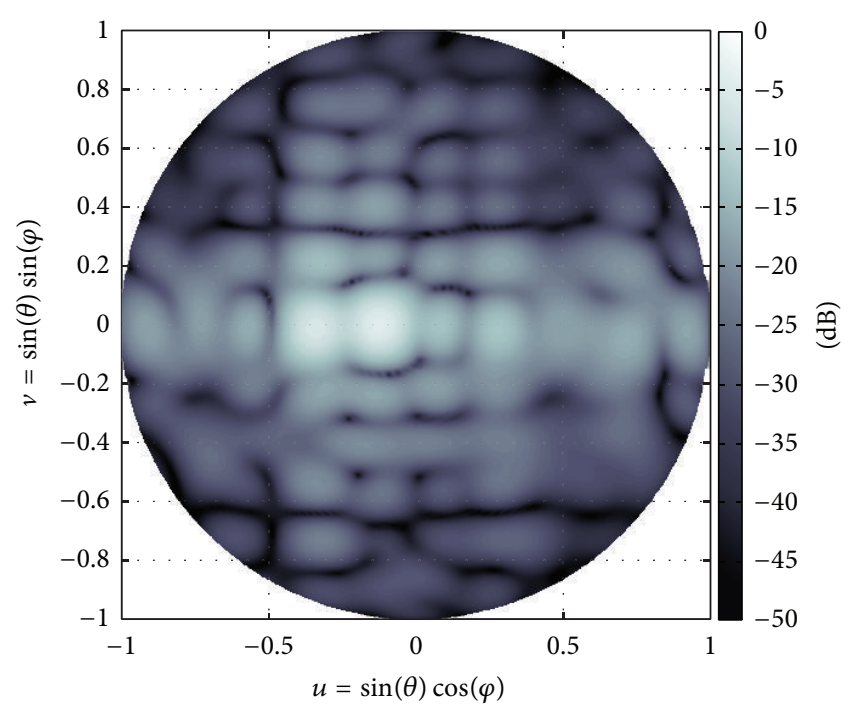

(a)

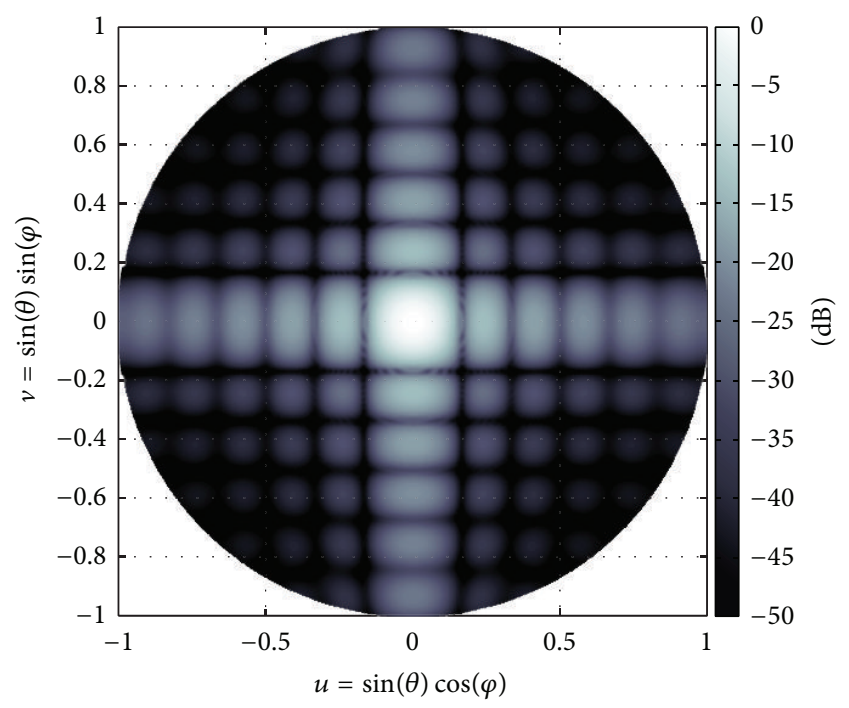

(b)

FIgURE 7: Simulated backscattering patterns under $y$-polarized normal incidence at $4 \mathrm{GHz}$ for (a) the nonuniform surface as instance and (b) the naked conducting surface. The far-field intensity involved in this figure is the synthesized quantity $\sqrt{\left|E_{\theta}\right|^{2}+\left|E_{\varphi}\right|^{2}}$. Both patterns are normalized to the specular far-filed intensity of the conducting surface and the values below $-50 \mathrm{~dB}$ have all been truncated.

surface in the band from 3.6 to $5.5 \mathrm{GHz}$ is suppressed by more than $10 \mathrm{~dB}$ and $7 \mathrm{~dB}$ for the $y$ - and $x$-polarized incident beams, respectively. Hence, the potential of the nonuniform surface to reduce RCS of conducting objects over a fairly wide frequency band is well demonstrated. The simulated reflectance is also shown in Figure 9 for comparison. We note that the measured reflectance minima appear at higher frequencies compared with their simulated counterparts. However, the measured and simulated reflectance curves are in qualitative agreement if we uniformly shift the measured curves towards lower frequency. The discrepancy between 


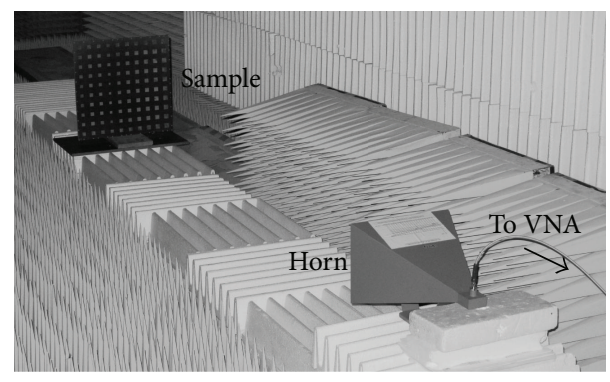

FIGURE 8: Experimental setup for measuring the specular reflectance of the nonuniform surface sample under normal incidence.

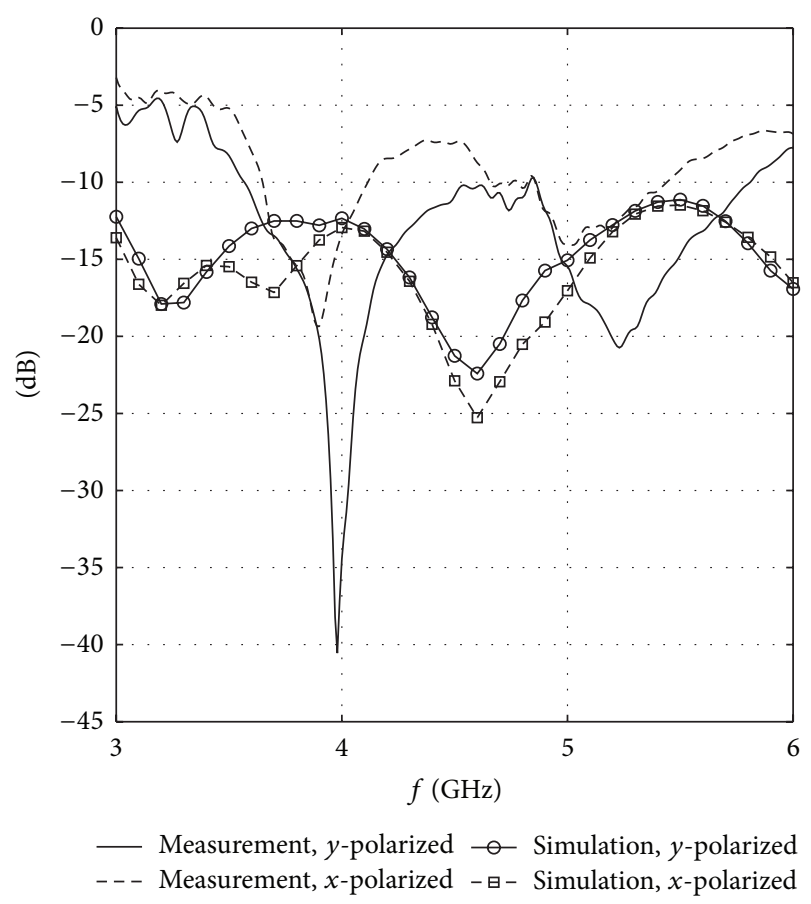

FIGURE 9: Experimental results of the normalized specular reflectance of the nonuniform surface sample with respect to both $x$ - and $y$-polarized normally incident waves. The $x$ and $y$ directions are indicated by Figure 5(a).

the simulated and measured results is mainly attributed to the inaccuracy of the substrate permittivity, the fabrication and assembly error of the sample, and the differences between the experimental setup and the simulation configuration. In our experimental setup, the sample is in the radiating near-field (Fresnel) region of the horn antenna and the local incident rays for most of the reflecting elements are just roughly perpendicular to the element surface. In other words, the final experimental reflectance is not in a strict far-field sense and most of the reflecting elements underwent oblique incidence in the experiment. Because the first-order resonant frequency of metallic patch increases as the incidence angle grows, the effective operating band of the metasurface moved to higher frequency region in the experiment.

\section{Conclusions}

We have demonstrated that planar metasurface with nonuniformly distributed reflection phase, referred to as nonuniform surface, is capable of suppressing the specular reflections of conducting plates by scattering most incident energy in off-specular directions. A general condition under which the nonuniform surface could operate effectively has been derived using a simplified theoretical model. Based on this condition, an instance of nonuniform surface composed of three-layer stacked-patch elements has been designed. Both simulation and measurement results for the design example confirm that the nonuniform surface is an effective approach of RCS-reduction for conducting objects and has the potential of providing broadband performance.

\section{Conflict of Interests}

The authors declare that there is no conflict of interests regarding the publication of this paper.

\section{Acknowledgments}

This work was supported in part by the Natural Science Foundation of Jiangsu Province under Grant BK20130326, in part by the National Natural Science Foundation of China under Grant 61301076, in part by the Open Research Program of State Key Laboratory of Millimeter Waves in China under Grant K201417, and in part by the Natural Science Foundation of the Higher Education Institutions of Jiangsu Province under Grant 12KJB510030.

\section{References}

[1] W. W. Salisbury, "Abs orbent body for electromagnetic waves," U. S. Patent 2599 944, June 1952.

[2] E. F. Knott, J. F. Shaeffer, and M. T. Tuley, Radar Cross Section, Artech House, London, UK, 1993.

[3] Y. Z. Ruan, Radar Cross Section and Stealth Technology, National Defence Industry Press, Beijing, China, 1998.

[4] J. A. Adam, "How to design an invisible aircraft," IEEE Spectrum, vol. 25, no. 4, pp. 26-31, 1988.

[5] N. Engheta, "Thin absorbing screens using metamaterial surfaces," in Proceedings of the IEEE Antennas and Propagation Society International Symposium, vol. 2, pp. 392-395, 2002.

[6] F. Costa and A. Monorchio, "Multiband electromagnetic wave absorber based on reactive impedance ground planes," IET Microwaves, Antennas and Propagation, vol. 4, no. 11, pp. 17201727, 2010.

[7] F. Costa and A. Monorchio, "A frequency selective radome with wideband absorbing properties," IEEE Transactions on Antennas and Propagation, vol. 60, no. 6, pp. 2740-2747, 2012.

[8] J. B. Pendry, D. Schurig, and D. R. Smith, "Controlling electromagnetic fields," American Association for the Advancement of Science: Science, vol. 312, no. 5781, pp. 1780-1782, 2006.

[9] D. Schurig, J. J. Mock, B. J. Justice et al., "Metamaterial electromagnetic cloak at microwave frequencies," Science, vol. 314, no. 5801, pp. 977-980, 2006.

[10] M. Paquay, J.-C. Iriarte, I. Ederra, R. Gonzalo, and P. de Maagt, "Thin AMC structure for radar cross-section reduction," IEEE 
Transactions on Antennas and Propagation, vol. 55, no. 12, pp. 3630-3638, 2007.

[11] R. Liu, C. Ji, J. J. Mock, T. J. Cui, and D. R. Smith, "Random gradient index metamaterials," in Proceedings of the International Workshop on Metamaterials, pp. 248-250, 2008.

[12] X. M. Yang, X. Y. Zhou, Q. Cheng, H. F. Ma, and T. J. Cui, "Diffuse reflections by randomly gradient index metamaterials," Optics Letters, vol. 35, no. 6, pp. 808-810, 2010.

[13] X. M. Yang, Q. Cheng, and T. J. Cui, "Research on the onedimensional randomly gradient index coating," in Proceedings of the International Conference on Microwave and Millimeter Wave Technology (ICMMT '10), pp. 1208-1211, Chengdu, China, May 2010.

[14] K. Wang, J. Zhao, Q. Cheng, D. S. Dong, and T. J. Cui, "Broadband and broad-angle low-scattering metasurface based on hybrid optimization algorithm," Scientific Reports, vol. 4, article 5935, 2014.

[15] R. H. Clarke and J. Brown, Diffraction Theory and Antennas, Ellis Horwood, Chichester, UK, 1980.

[16] C. S. Malagisi, "Microstrip disc element reflect array," in Proceedings of the Electronics and Aerospace Systems Convention (EASCON'78), pp. 186-192, 1978.

[17] J. P. Montgomery, "A microstrip reflectarray antenna element," in Proceedings of the Antenna Applications Symposium, University of Illinois, Urbana, Ohio, USA, 1978.

[18] D. M. Pozar and T. A. Metzler, "Analysis of a reflectarray antenna using microstrip patches of variable size," Electronics Letters, vol. 29, no. 8, pp. 657-658, 1993.

[19] J. Huang and J. A. Encinar, Reflectarray Antennas, John Wiley \& Sons, Hoboken, NJ, USA, 2007.

[20] J. A. Encinar, "Design of two-layer printed reflectarrays using patches of variable size," IEEE Transactions on Antennas and Propagation, vol. 49, no. 10, pp. 1403-1410, 2001.

[21] J. A. Encinar and J. A. Zornoza, "Broadband design of threelayer printed reflectarrays," IEEE Transactions on Antennas and Propagation, vol. 51, no. 7, pp. 1662-1664, 2003. 

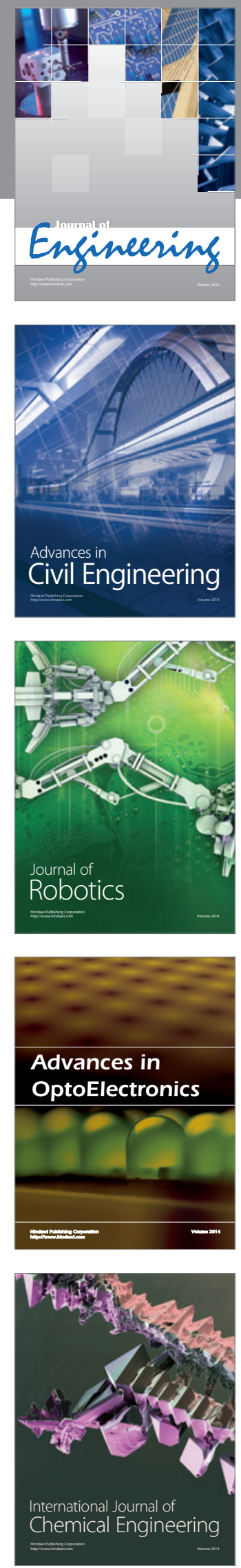

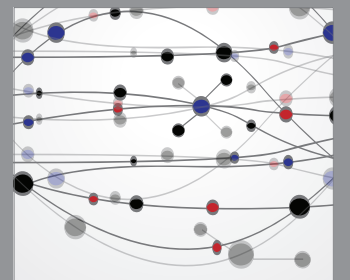

The Scientific World Journal
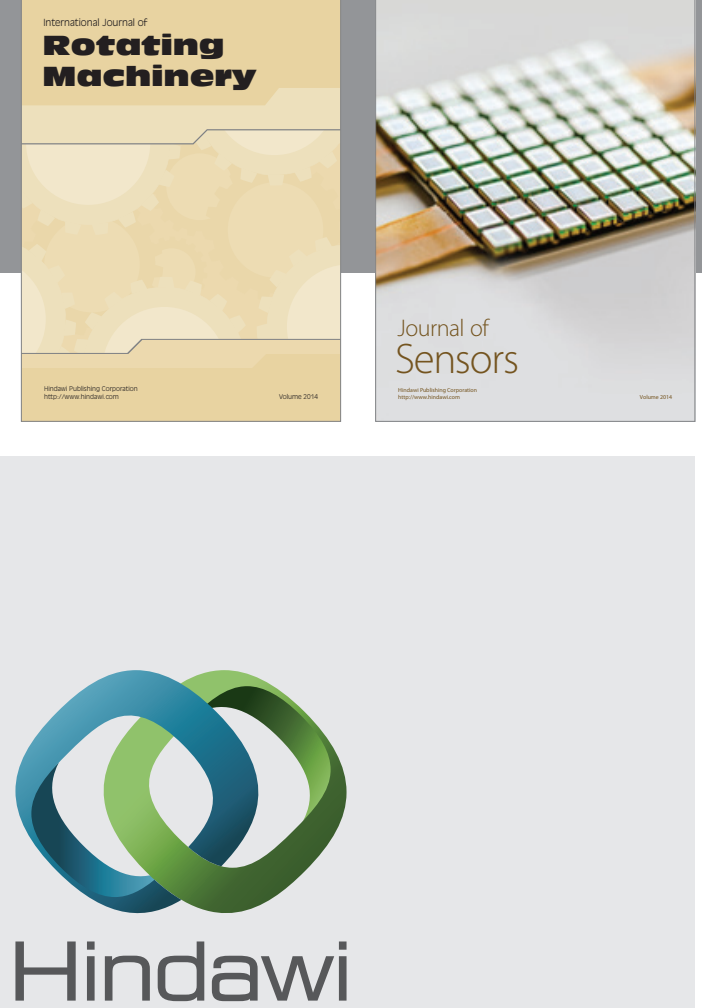

Submit your manuscripts at http://www.hindawi.com
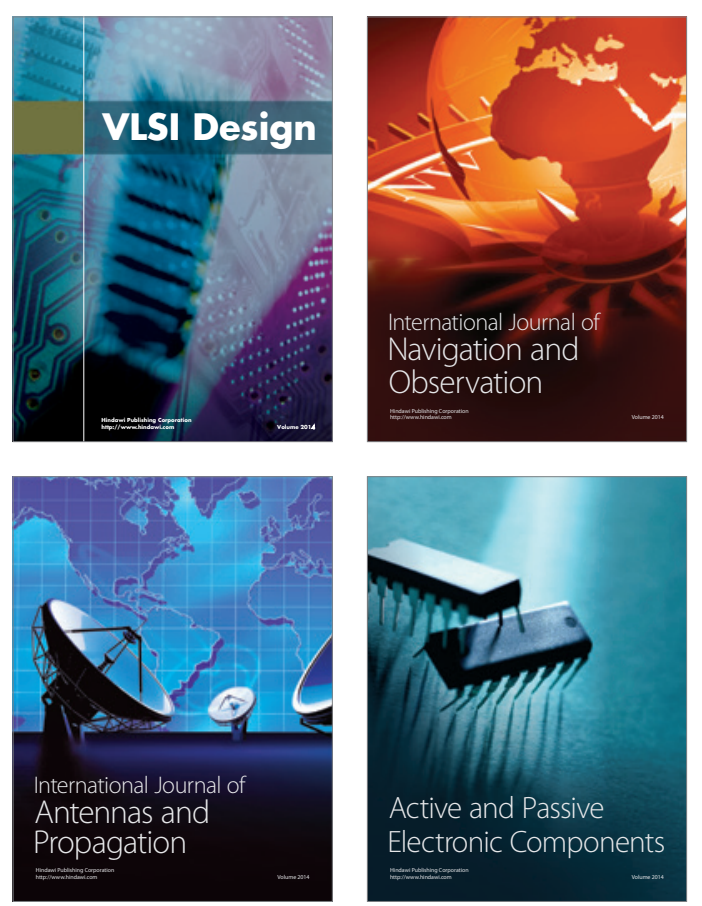
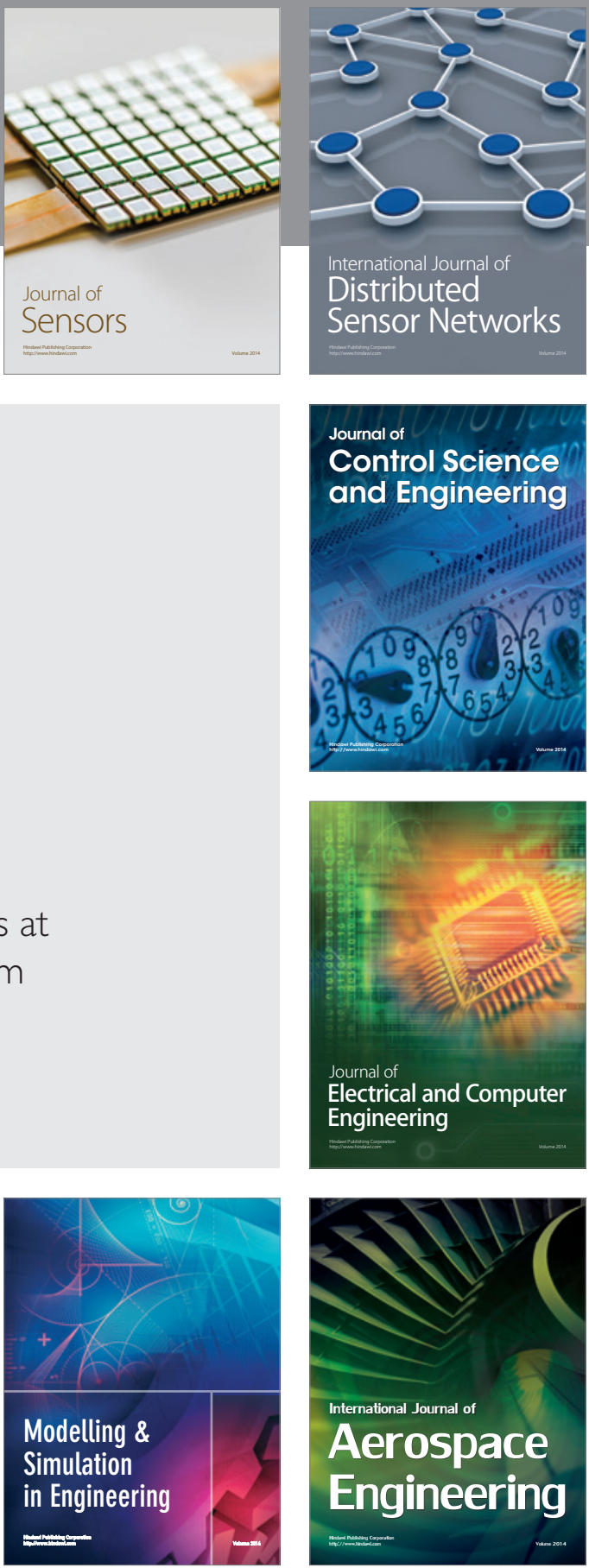

Journal of

Control Science

and Engineering
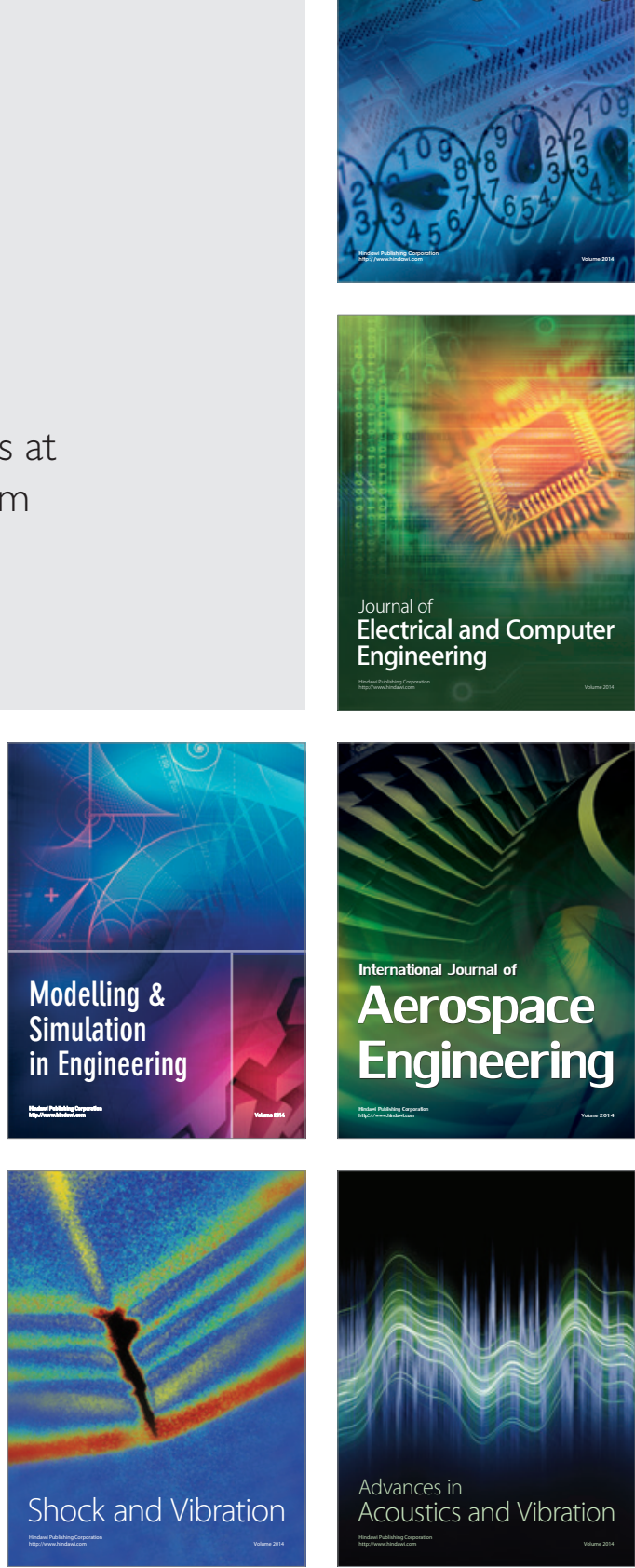